\title{
Deploying Clouds in the Guifi Community Network
}

\author{
Roger Baig*, Jim Dowling ${ }^{\S}$, Pau Escrich*, Felix Freitag ${ }^{\dagger}$, Roc Meseguer ${ }^{\dagger}$, Agusti Moll*, Leandro Navarro ${ }^{\dagger}$, Ermanno Pietrosemoli ${ }^{\ddagger}$, \\ Roger Pueyo*, Vladimir Vlassov ${ }^{\top}$, Marco Zennaro $\ddagger$ \\ *Fundació Privada per la Xarxa Lliure, Oberta i Neural Guifi.net. Mas l'Esperana, 08503 Gurb, Catalonia \\ \{roger.baig, pau.escrich, agusti.moll, roger.pueyo\}@guifi.net \\ $\dagger$ Department of Computer Architecture. Universitat Politècnica de Catalunya. Barcelona, Spain \\ \{felix, leandro\}@ac.upc.edu \\ ${ }^{\ddagger}$ The Abdus Salam International Centre for Theoretical Physics (ICTP). Trieste, Italy. \\ \{ermanno, mzennaro\}@ictp.it \\ $\S$ The Swedish Institute of Computer Science (SICS). Stockholm, Sweden. \\ \{jdowling\}@sics.se \\ 『KTH Royal Institute of Technology. Stockholm, Sweden. \\ $\{$ vladv\}@kth.se
}

\begin{abstract}
This paper describes an operational geographically distributed and heterogeneous cloud infrastructure with services and applications deployed in the Guifi community network. The presented cloud is a particular case of a community cloud, developed according to the specific needs and conditions of community networks. We describe the concept of this community cloud, explain our technical choices for building it, and our experience with the deployment of this cloud. We review our solutions and experience on offering the different service models of cloud computing (IaaS, PaaS and SaaS) in community networks. The deployed cloud infrastructure aims to provide stable and attractive cloud services in order to encourage community network user to use, keep and extend it with new services and applications.
\end{abstract}

Index Terms-community networks; cloud computing;

\section{INTRODUCTION}

Hundreds of community networks operate across the globe, in rural and urban, rich and poor areas. Networks exist almost everywhere e.g. in the USA, WasabiNet is running in St. Louis, Missouri while The Personal Telco Project is deployed in Portland, Oregon. In Latin America, numerous networks exist and cooperate, e.g. Bogota Mesh and Montevideo Libre. For instance in Melbourne, Australia, Melbourne Wireless is a quickly growing community network. In Europe, FunkFeuer in Austria, AWMN in Athens, Greece, Guifi.net in Spain and Freifunk in Germany have reached thousands of nodes.

Community networks, also known as bottom-up-broadband networks, are a communication infrastructure in which local communities of citizens build, operate and own open IP-based networks. Community networks often originated as a solution for providing Internet access to the population of areas which were unattended by commercial telecom operators.

Cloud computing is nowadays a cost-effective, elastic and scalable way to build and support Internet-based applications. In the areas where Internet access and bandwidth is provisioned, the wide offer of commercial cloud solutions has led to a widespread adoption of cloud usage by all kinds of stakeholders.
Within community networks, however, the possibilities of cloud computing have not yet been exploited. The community cloud we present in this paper aims to materialize the vision of a cloud for community networks, hosted on community-owned computing and communication resources providing services of local interest.

In general, a community cloud is a cloud deployment model in which a cloud infrastructure is built and provisioned for use by a specific community of consumers with shared concerns, goals and interests, and is owned and managed by the community or by a third party or a combination of both [1]. A community cloud therefore offers features that are tailored to the needs of a specific community. The cloud deployment experience that we present in this paper is on a particular case of a community cloud, a cloud for community wireless networks. While the foundational elements of such a community cloud solution have been discussed before [2], a successful implementation and deployment has not been reported yet.

The contributions of this experience paper are the followings:

1) The technical choices that we adopted for deploying a real cloud system in a community wireless network.

2) Design decisions specific for this community cloud, such as the Cloudy distribution.

3) Experiences with a heterogeneous cloud system built with off-the-shelf devices and using popular cloud management platforms.

The structure of this paper is as follows: In section 2 we introduce the concept of clouds in community networks in order to explain the context we address. In section 3 we sketch the overall architecture of the community cloud. We explain in section 4 the platforms and approach we used to build the real community cloud, with reference to the deployed cloud system. In section 5 we discuss our current results and conclude the paper in section 6 . 


\section{CONCEPT}

The community cloud that we present in this paper needs to address the conditions of community networks and satisfy their requirements. The following analysis reviews such requirements and the scenarios of these community clouds to understand better how they could fit into networks built by people.

Topology of community networks and hardware aspects: A community wireless network distinguishes between super nodes, also called backbone nodes, and client nodes. Super nodes have at least two wireless links, each to other super nodes. Super nodes are installed in the community network participants premises or are strategically placed on a third party location, e.g. telecommunication installations of municipalities, to improve the community network backbone. Client nodes only connect to a super node, but do not route any traffic. A super nodes in a community network is typically assigned an IP address range, e.g. a /27 range, which are public within the community network ${ }^{1}$.

Cloud resources therefore should be attached to super nodes in order to obtain a public IP address which allows them to easily offer public services.

In general, the hardware used in community networks for routers and antennas is heterogeneous and off-the-shelf. The same applies for servers for network management or end-user services.

As a consequence, our community cloud must be able to operate with heterogeneous hardware.

Social aspects of community networks: Personal and social relationships play an important role in the community wireless network deployment. The deployment of new nodes needs the collaboration among people. Participants of community networks are consumers and producers of the network. Most of them as producers contribute infrastructure and time to the network. As consumers, they use the available services and the network bandwidth. However a community network does not work solely from the contribution of infrastructure. Some users must also contribute time and knowledge. Contribution of time is needed for instance for maintenance tasks (tasks which might require technical knowledge or not). Technical knowledge is needed because the network is typically is an IP network, which needs to be managed and configured.

As a consequence, the community cloud needs to be collectively built and maintained, which can be seen as an extension of the collective and shared effort upon which community networks are already built, but aimed towards services and applications.

Ownership of nodes in community networks: Typically, when a new member wants to connect to the community network, it contributes with the hardware required to connect to other nodes. In such case the new node of the community network belongs to the member who is its sole owner. Another option is that a group of people team up to jointly

\footnotetext{
${ }^{1}$ We refer to routable IP addresses within the community network which are not behind a NAT.
}

crowd-fund a node. Examples for crowd-funding are backbone nodes that connect one wireless network area with another. In principle both options, individual ownership and crowdfunding of resources, could be considered as way to contribute infrastructure to community clouds.

In the design of our community cloud, the different possible ownership modes need to be taken into account for the contribution and administration of the cloud infrastructure.

Given these socio-technical characteristics of community wireless networks, we consider in Figure 1 how the community cloud could fit into a typical community wireless nework. The picture shows some community nodes with a router and some servers attached to it. Some clients nodes are shown that are connected to the access point (AP) of a super node. In addition, however, cloud resources are attached to some super nodes. These cloud resources are part of the community cloud. Furthermore, depending on the topology of the community network and its social structure, several local community clouds may appear which manage locally the cloud resources belonging to a certain zone defined geographically or by its social networks. We will call such local clouds microclouds. A set of microclouds are interconnected then through the wireless backbone network.

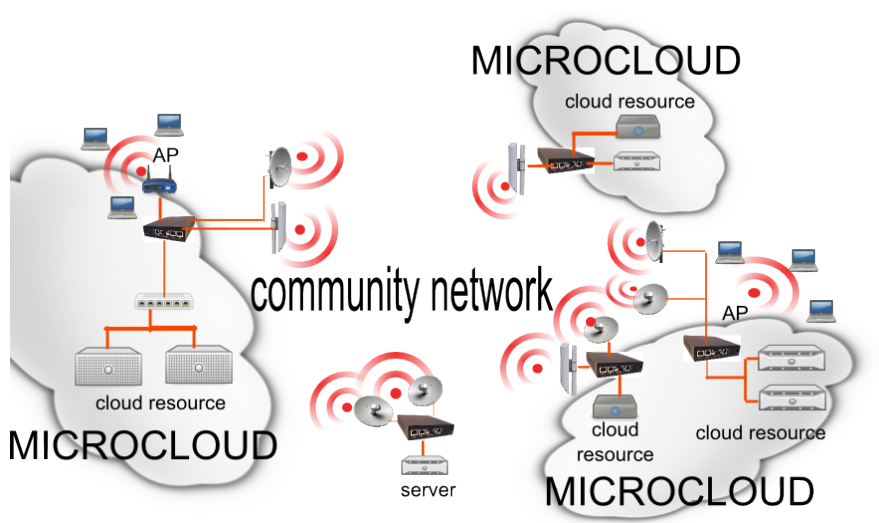

Fig. 1. Microclouds in a community network

\section{OVERALL ARCHITECTURE}

The following overall architecture organizes the different layers of the cloud system for the development of the cloud system in community networks. Figure 2 shows a high-level layered view that integrates the users, software, hardware and the network. From a top-down perspective, starting with the end user and going down to the hardware components and community network, the community cloud has the following layers:

- User interface and applications: End users of the community network are provided with applications and user interfaces to interact in a transparent way with the underlying community services.

- Services: Cloud-based services are provided by a software distribution which is hosted on the cloud infrastructure. 


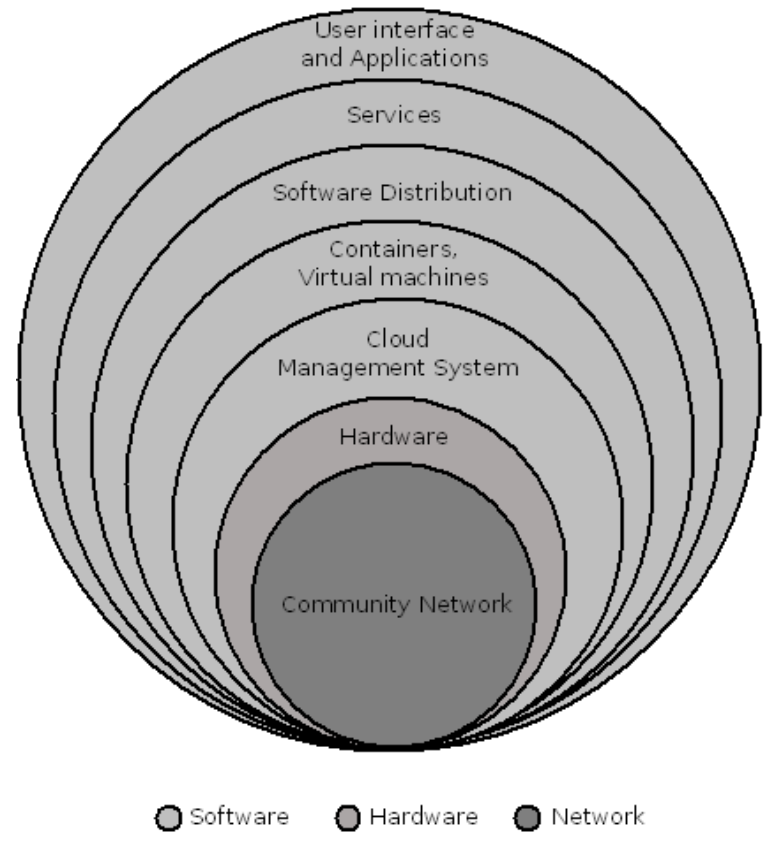

Fig. 2. Network and services in a cloud for community networks.

Service discovery and distributed storage are examples of these services.

- Software Distribution: The software distribution runs on the underlying infrastructure and hardware layers, i.e. it runs in the containers or virtual machines. Using a software distribution provides a way to pack and distribute a common set of cloud platform services.

- Containers and Virtual Machines: The elements of this layer are containers or virtual machines provided by the infrastructure service. They are created by the cloud management platform. The virtual machines are the environment where the software distribution is installed.

- Cloud Management Platform: This layer contains the software needed to manage the cloud platform. It coordinates the underlaying hardware layer with the software distribution layer using standard Linux tools and manages the containers and virtual machines layer.

- Hardware: This layer represents the cloud hardware layer. Given the heterogeneity of devices in community networks, this hardware can range from high-end servers to low-end resource-constraint home gateways. The hardware is connected to the community network through the network nodes.

- Community Network: This is the community network within which the community cloud is deployed.

\section{Deployed Community Cloud}

We describe in this section the cloud infrastructure that we have deployed in the Guifi community network. It is a real system and we explain how the layers described in the previous section are implemented, materializing the concept of the community cloud presented in section II.

\section{A. Community Network}

Guifinet $^{2}$ is our target community network where we deployed the cloud infrastructures. Among the community networks mentioned in the introduction section, Guifi can be considered the largest one worldwide with more than 20000 nodes. Figure 3 shows the wireless links and nodes of the Guifi.net in the area around Barcelona.

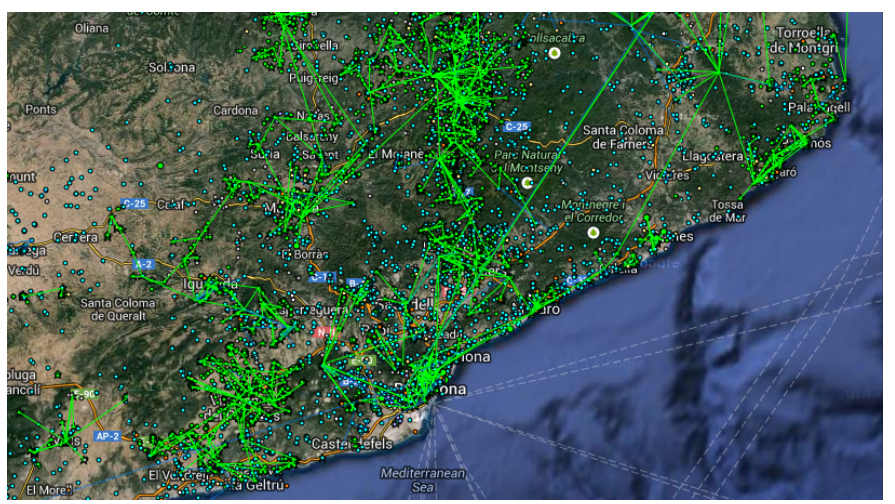

Fig. 3. Guifi.net nodes and links in the area around Barcelona.

\section{B. Hardware}

In order to correspond to the conditions of community networks, we deployed heterogeneous hardware to form the cloud infrastructure. A few nodes are high-end rack-based servers (PowerEdge R420 rack server), some cloud nodes are Dell OptiPlex 7010 desktops. These nodes support virtualization by hardware. In addition, however, we deployed cloud nodes that represents the case of low-end cloud resources such as home gateways, that users may provide to the cloud. Such kind of devices have been deployed through Community$\mathrm{Lab}^{3}$ [3][4] and mainly consist of small Jetway device (no support for KVM, only containers) Towards the lower end devices, we deployed some Alix boards and Intel Galileo boards for evaluation purposes. The current status of the cloud deployment can be seen in the Clommunity project's Wiki ${ }^{4}$.

\section{Cloud Management Platform}

For the management of the community cloud infrastucture we mainly used Proxmox ${ }^{5}$ and OpenStack ${ }^{6}$ as CMP. The reason for using Proxmox is that within the community network, there was already some positive usage experience, and the installation and operation of Proxmox is relatively easy compared to other CMPs. OpenStack on the other hand, is popular as a powerful customizable cloud platform, supported by a large user community, though not within community networks. Figure 4, shows the Proxmox administrator interface of a cluster of five of our Proxmox cloud nodes deployed in different locations of Guifi.net.

\footnotetext{
${ }^{2}$ http://guifi.net/

${ }^{3}$ http://community-lab.net/

${ }^{4}$ http://wiki.clommunity-project.eu/testbed:start

${ }^{5}$ http://www.proxmox.com/

${ }^{6} \mathrm{hhttp}: / /$ www.openstack.org/
} 


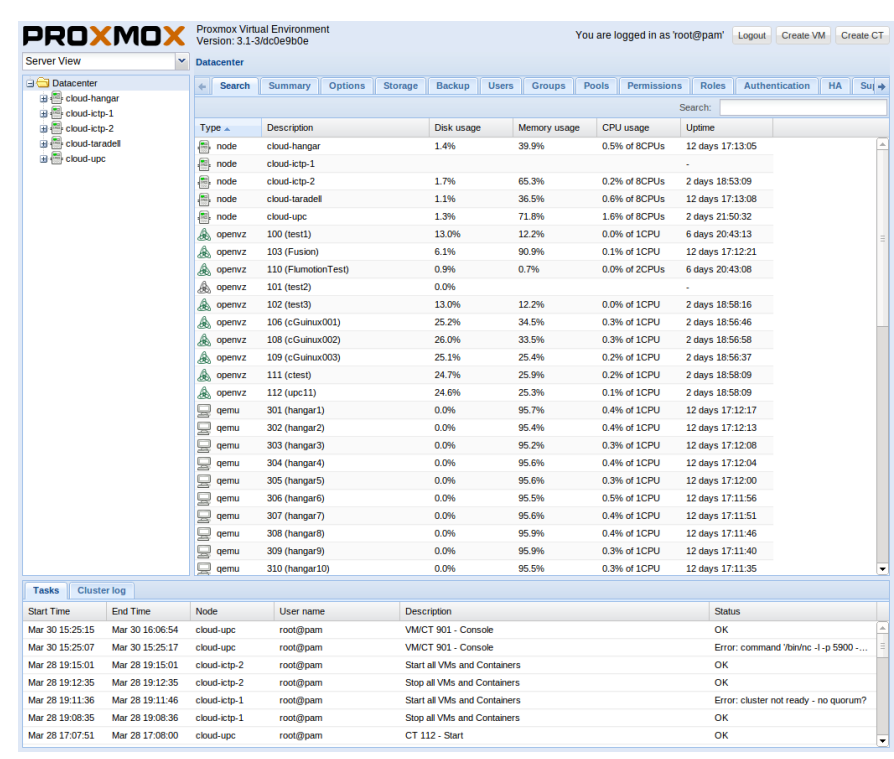

Fig. 4. Guifi cloud Proxmox VE cluster.

\section{Containers and Virtual Machines}

Cloud management platforms (CMPs) provide Infrastructure-as-a-Service (IaaS) in terms of virtual machines (VMs) and virtual networks. In our community cloud, the VMs in the higher-end cloud nodes are KVM and are managed by OpenStack and Proxmox CMPs. In the lower-end cloud nodes Linux containers are used and manged through Community-Lab ${ }^{7}$ [3] [4]. Smokeping is used for monitoring of the basic parameters of the cloud hardware with publicly available view ${ }^{8}$.

\section{E. Software Distribution}

We have developed a community cloud GNU/Linux distribution, codenamed Cloudy, aimed and designed for building clouds in community networks. This distribution contains the platform and application services of the community cloud model.

Cloudy $^{9}$ is the core of our cloud system, because it unifies the different tools and services of the cloud system in a Debian-based Linux distribution. Each community network user that contributes infrastructure to the cloud is encouraged to install the Cloudy distribution. In Figure 5 the installation screen of the Cloudy distribution is shown. Cloudy installs like a standard Debian distribution and is given in three flavors: As a standalone version to install on real hardware, a containerbased one to install on a virtualized operating system, and an operating system image to install on virtual machines.

Cloudy aims to be deployed on the cloud resources that form the microcloud in the community network, as illustrated in Figure 6. Each cloudy instance has a Web-based GUI, see Figure 7, for the cloud node administrator to install and

\footnotetext{
${ }^{7}$ http://community-lab.net/

${ }^{8} \mathrm{http} / / /$ monitor.clommunity-project.eu/smokeping

${ }^{9} \mathrm{http} / / /$ repo.clommunity-project.eu/
}

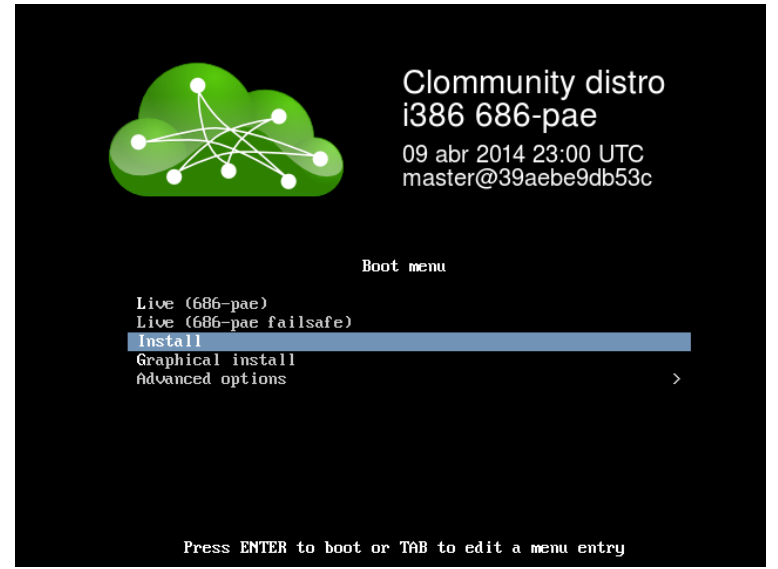

Fig. 5. Installing the Cloudy distribution.

configure in an easy and comfortable way cloud application services and community network services.

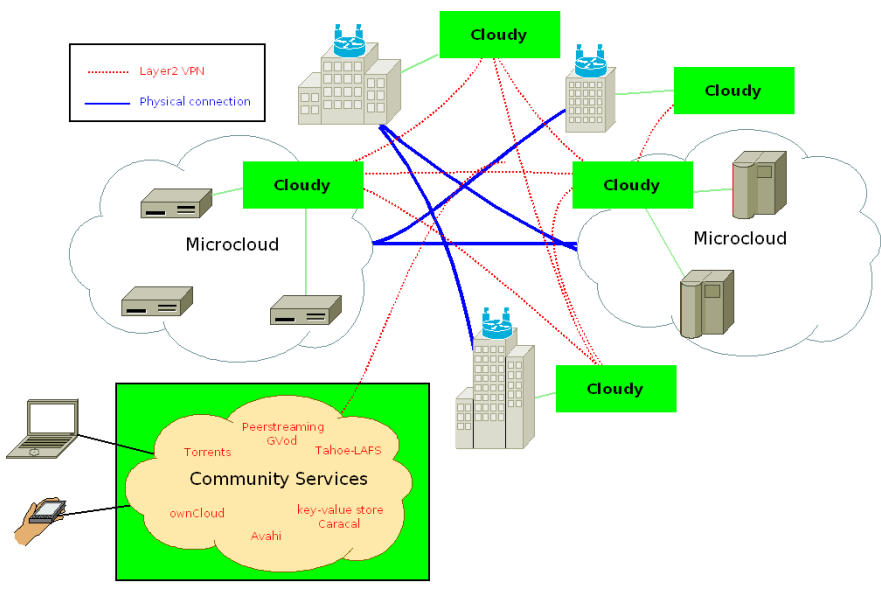

Fig. 6. Cloudy distribution deployed in microclouds.

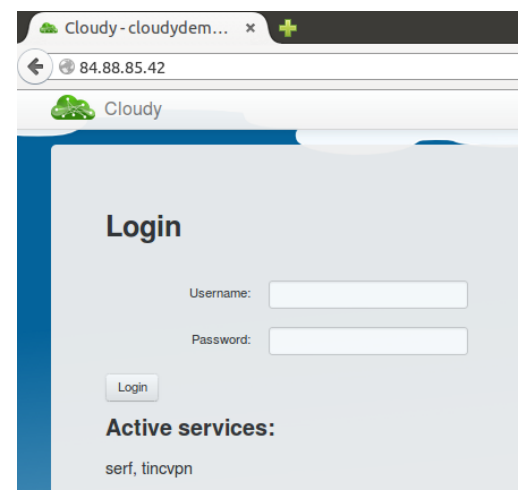

Fig. 7. Cloudy login screen used by cloud node owner.

\section{F. Services}

We provide in the Cloudy distribution a set of pre-installed services, which community network users are expected to 
find useful and attractive, grouped as Search, Clommunity, and Guifi.net. The Search service allows the user to find Cloudy instances in the community cloud, and discover services deployed in these Cloudy instances. Different search options are available (Figure 8). Depending on the chosen search mechanism, search is done over the instances of the microcloud or over all Cloudy instances. Figure 9 shows services in other Cloudy instances discovered by the search service.

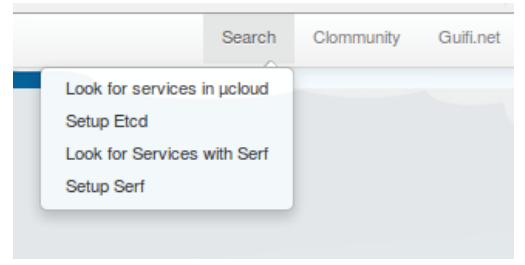

Fig. 8. Discover instances and services in the community cloud.

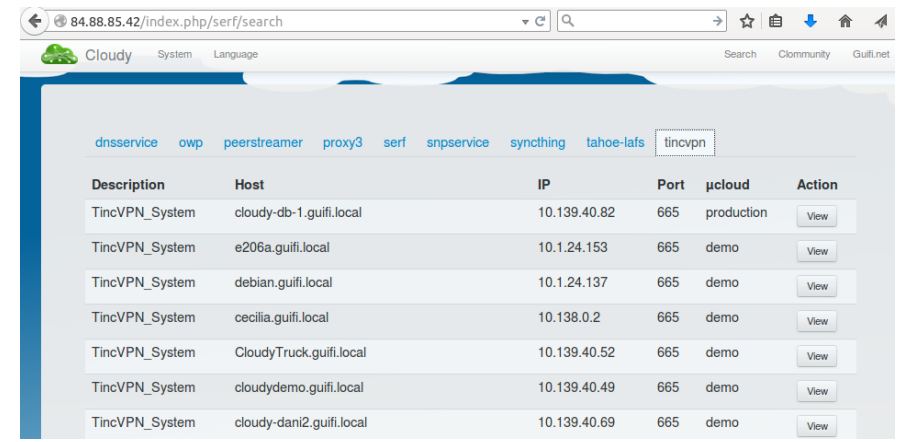

Fig. 9. Cloudy instances discovered by the Search service.

The Clommunity service menu in the Cloudy GUI shows the application which come already integrated in the cloud distribution (although the user is free to decide if he/she wants to activate them), see Figure 10. In the current version of Cloudy, Tahoe-LAFS as a service that allows building secure storage is already integrated. For video streaming, we currently study different options of Peer-to-Peer based platforms.

\begin{tabular}{l|}
\hline Search Clommunity \\
\hline Getinconf \\
OpenVZ Web Panel \\
PeerStreamer \\
Syncthing \\
Tahoe-LAFS \\
WebDAV server \\
\hline
\end{tabular}

Fig. 10. Application services offered by Cloudy.

The Guifi.net services allow to install a set of community network management services (Figure 11). These services include a proxy service based on Squid, usually used to enable Internet access from within the community network, a SNMP service for network monitoring, and a DNS service for name resolution within the community network.

\begin{tabular}{|c|c|c|}
\hline Search & Clommunity & Guifi.net \\
\hline & \multicolumn{2}{|l|}{ Proxy3 } \\
\hline & \multicolumn{2}{|c|}{ SNPServices } \\
\hline & \multicolumn{2}{|c|}{ DNSServices } \\
\hline
\end{tabular}

Fig. 11. Community network management services offered by Cloudy.

If the user activates Tahoe-LAFS, a robust distributed file system with end-to-end encryption will become available. At the client side, it combines security with erasure-coding on the objects, which allows to recover stored data even under the conditions of massive storage server failures. Figure 12 shows the GUI of the Tahoe-LAFS introducer with several storage servers provided by several of the deployed cloud nodes.

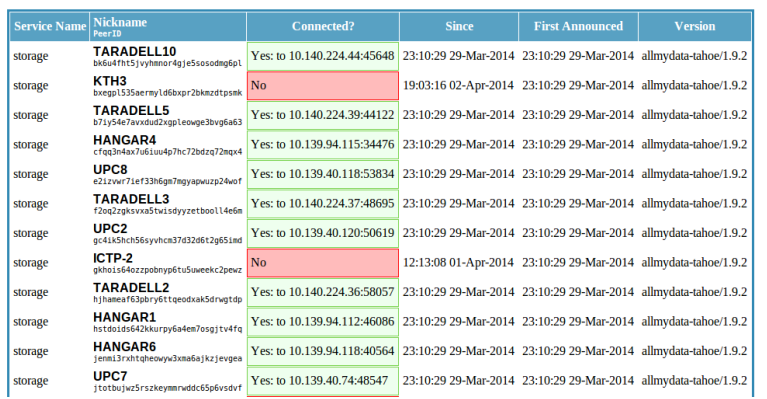

Fig. 12. Tahoe-LAFS introducer page.

\section{G. UI and applications}

In Figure 13 the user interface of the ownCloud application is shown. OwnCloud offers some functionalities which are similar to Dropbox, a commercial cloud-based storage solution, but ownCloud is open source. We have started experimenting with integrating ownCloud in Cloudy since ownCloud is popular within community network users.

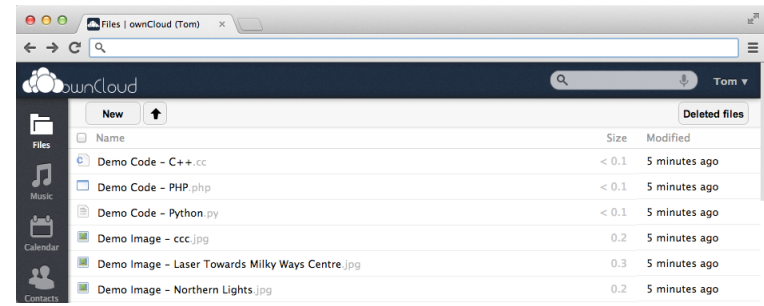

Fig. 13. Owncloud user interface. 


\section{DISCUSSION}

After presenting in the previous section the technical choices we adopted to build the community cloud, we discuss in this section some results which we obtained from deploying cloud nodes in Guifi.

\section{A. Deployment experience}

We achieved deploying an important number of cloud nodes in the Guifi.net ${ }^{10}$, thanks to the collaboration of community networks members with this project. Heterogeneous hardware was used to build the cloud infrastructure. We found the need to use different CMPs to provide IaaS since our community cloud also includes low resource nodes with Linux containers, which were not well or not at all supported by OpenStack and Proxmox, respectively.

The different options for searching other Cloudy instances and cloud services obtain the required information. Global search is mainly conducted while the search limited to within the microcloud will gain importance once the number of deployed local clouds has increased. We are currently experimenting with providing a service quality measure along with the discovered services, in order to help the user the selection among the found services.

We conducted some experiments on the performance of applications deployed in our cloud. The results are detailed in [5] and showed satisfactory performance, suggesting their usefulness for end users.

The Cloudy distribution is available for download ${ }^{11}$. Users can extend it with new services, either as contribution to the Cloudy source or as deployed services.

\section{B. Extending geographic reach}

Growth of a community wireless network is typically limited to a local area reachable through wireless links. For the service level, which we address with community clouds, this geographic limitation does no longer hold. We have added cloud nodes over Internet tunnels to the community network, i.e. with Guifi IP range and routable, in distances beyond any wireless link, hundreds of kilometers ways, such as nodes in Stockholm ${ }^{12}$, Sweden and Trieste ${ }^{13}$, Italy.

\section{Sustainability}

As mentioned before, the ultimate goal of the deployment of this community cloud is the uptake of cloud-based services in community networks by their users. Our approach includes several measures to achieve this user uptake: 1) We provide already integrated in Cloudy a basic set of potentially attractive applications. 2) Each user which installs Cloudy may also activate the basic services, therefore it is likely there will always be a minimum set of nodes active which are needed to make the cloud services available. 3) The Cloudy distribution

\footnotetext{
${ }^{10} \mathrm{~A}$ public cloudy instance is currently available at http://84.88.85.42:7000/ which allows to obtain a live view on the community cloud nodes.

${ }^{11} \mathrm{http}: / /$ repo.clommunity-project.eu/images/

${ }^{12}$ https://guifi.net/node/63332

${ }^{13}$ https://guifi.net/node/63828
}

includes also the most needed services to manage the community network itself and in an improved user-friendly way, which makes Cloudy an attractive option for installers of new nodes.

\section{CONCLuSion}

This paper presented a cloud deployed in a community network for providing services and applications to the users. Such deployment benefited from the consolidation of cloud technology, which enabled to provide IaaS through popular CMPs. Additional efforts were carried out to include also low-resource cloud nodes, in which Linux containers were managed, leading to a very heterogeneous cloud system. The presented cloud system is a community cloud whose technical choices and deployment were tailored to the particular conditions of community wireless networks.

The approach to provide PaaS and SaaS is materialized in the Cloudy distribution. This Debian-based Linux distribution integrates a set of services which through Avahi-Tinc enable the discovery of other Cloudy instances and their services.

All software needed to build the presented community cloud is available in public repositories, both in source code and as installable images, with the goal of encouraging users to install additional cloud nodes.

The users can add additional services to their cloud nodes. Combined with the possibility to run Cloudy on different hardware ranging from high-end to low-resource devices, we can expect that by having made user contributions easy, new user-driven cloud-based services, e.g. from the IoT domain, will appear in this community cloud in the future.

\section{ACKNOWLEDGMENT}

This work was supported by the European Framework Programme 7 FIRE Initiative projects CONFINE (FP7288535), CLOMMUNITY (FP7-317879), Universitat Politcnica de Catalunya-BarcelonaTECH and by the Spanish government under contract TIN2013-47245-C2-1-R.

\section{REFERENCES}

[1] "The NIST Definition of Cloud Computing". National Institute of Science and Technology, U.S. department of commerce." [Online]. Available: http://csrc.nist.gov/publications/nistpubs/800-145/SP800-145.pdf

[2] A. Marinos and G. Briscoe, "Community Cloud Computing," Computing, vol. 5931, no. December, p. 11, Jul. 2009.

[3] B. Braem and et al., "A case for research with and on community networks," ACM SIGCOMM Computer Communication Review, vol. 43, no. 3, pp. 68-73, Jul. 2013.

[4] M. Selimi, J. Florit, D. Vega, R. Meseguer, E. Lopez, A. M. Khan, and et al., "Cloud-based extension for community-lab," in 22nd International Symposium on Modeling, Analysis and Simulation of Computer and Telecommunication Systems (MASCOTS14).

[5] M. Selimi, F. Freitag, R. Pueyo, and A. Moll, "Distributed storage and service discovery for heterogeneous community network clouds," in 7 th IEEE/ACM International Conference on Utility and Cloud Computing (UCC 2014). 